\title{
Clinical features and repair of ventricular septal defect and left ventricular aneurysm complicating myocardial infarction
}

\author{
A. D. Heath, A. M. Harris, and M. P. Wright \\ From Departments of Cardiology and Thoracic Surgery, St. George's Hospital, London S.W.I
}

A 52-year-old man developed a ventricular septal defect and ventricular aneurysm after myocardial infarction. The two defects were repaired through the left ventricular aneurysm using hypothermic techniques. Only two similar cases have been previously reported.

Myocardial infarction is frequently complicated by the development of ventricular lesions, the commonest being a ventricular aneurysm followed by ventricular septal defect and ruptured papillary muscles. These acquired lesions are all amenable to surgical repair, and this has become more common in recent years.

The first successful repair of an acquired ventricular aneurysm was made by Beck in I944 using a fascia lata graft to plicate the aneurysm. Acquired ventricular septal defect was first surgically repaired by Cooley $e t a l$. (1957) using cardio-pulmonary bypass and hypothermia. Subsequently similar acquired lesions were corrected surgically on many occasions, but not until 1962 were both defects repaired at the same operation. This case report describes the successful repair of an acquired ventricular septal defect and left ventricular aneurysm through the left ventricular aneurysm.

\section{Case report}

An exhibition erector, a man of 52, was admitted to St. George's Hospital, London, on 4 November 1967. Thirty-six hours previously he had suffered a sudden attack of vomiting, followed by dyspnoea and central chest pain which radiated through to the back. For several months he had experienced angina of effort.

On examination he was breathless at rest, with central cyanosis. Pulse 128/minute regular, jugulovenous pressure $+6 \mathrm{~cm}$. water, blood pressure $120 / 85 \mathrm{~mm}$. $\mathrm{Hg}$.

The heart sounds were normal, but there was a loud pansystolic murmur at the left sternal edge. There were bilateral basal crepitations, the liver was firm and palpable, but there was no peripheral oedema.
The electrocardiogram (Fig. I) showed an inferior infarct, and the chest $x$-ray (Fig. 2a) showed some cardiac enlargement and bilateral pulmonary oedema.

On the evening of admission he became shocked and there was wide splitting of the second sound in the pulmonary area, the systolic murmur was louder, and there was a pericardial rub. The diagnosis was thought to be either mitral incompetence from a ruptured chorda or ventricular septal defect as a result of an acute myocardial infarct.

Bed-rest and conservative management with digoxin and diuretics produced a good response and over the next three weeks there was steady improvement though the pansystolic murmur persisted and the second heart sound was widely split in expiration (Fig. 3a).

By 5 December plans had been made for his discharge to await cardiac catheterization in one to two months, but that morning he developed acute left ventricular failure which responded rapidly to intravenous frusemide and morphine. It was now felt that he should undergo immediate cardiac catheterization with a view to surgical correction of his myocardial lesion.

Cardiac catheterization and angiocardiography were performed on 8 December. A torrential leftto-right shunt was demonstrated by serial blood samples at ventricular level and the pulmonary artery pressure was $50 / 25 \mathrm{~mm}$. $\mathrm{Hg}$, pulmonary capillary mean pressure was $16 \mathrm{~mm}$. $\mathrm{Hg}$, and the left ventricular end-diastolic pressure was 5 $\mathrm{mm}$. Hg. Biplane Elema angiogram, with injection of contrast medium into the left ventricle, showed the mitral valve to be competent, and confirmed the left-to-right shunt at ventricular level (Fig. 2b). The diaphragmatic surface of the left ventricle was seen to move paradoxically on $x$-ray screening, indicating a ventricular aneurysm. Median sternotomy was started shortly after the cardiac investigation was completed. A thin fibrous aneurysm was seen over the diaphragmatic surface of the left ventricle and there was a coarse 


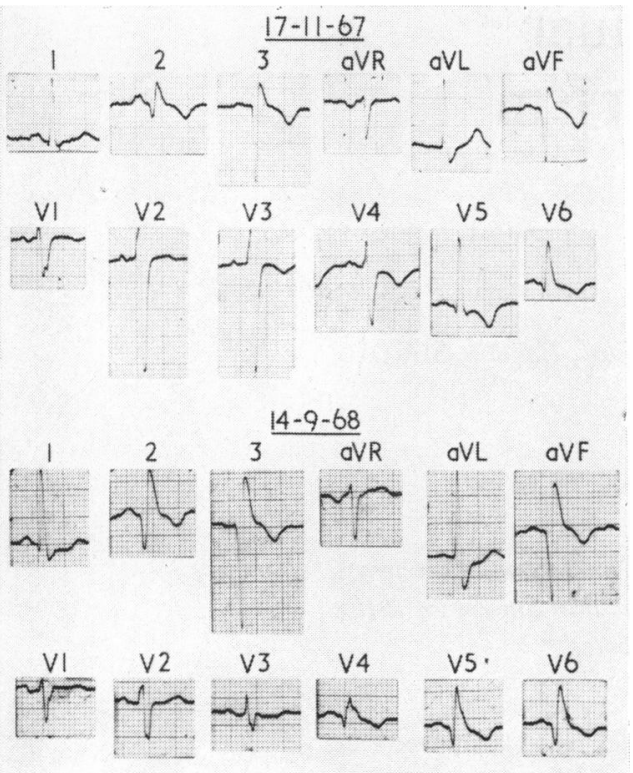

FIG. I Above. Pre-operative electrocardiogram showing $Q$ waves in leads II, III, aVF, V5-V6. Below. Electrocardiogram recorded Io months after operation showing very little change from the pre-operative recording.

FIG. 2a Pre-operative chest $\mathrm{x}$-rays showing cardiac enlargement and pulmonary venous congestion.

FIG. $2 \mathrm{~b}$ Elema angiogram with injection of Angioconray into the left ventricular cavity, showing simultaneous filling of the left and right ventricles through the ventricular septal defect and an inferior left ventricular aneurysm.

FIG. 2c Chest x-ray obtained ro months after operation showing a reduction in heart size and normal lung fields. thrill palpable anteriorly over the septum which could be abolished by local pressure.

Right and left heart bypass circuits were established without an oxygenator, and the blood was cooled to $14^{\circ} \mathrm{C}$. by heat exchanger in the line returned to the femoral artery.

The pumps were stopped and the still heart was dislocated forwards to expose the aneurysm. The fibrous area was incised towards the apex of the heart, and on examining the interior of the left ventricle a low septal defect was found communicating by multiple holes with the trabeculae of the right ventricle. Repair was effected with Terylene sutures tied over Teflon felt buttresses. After excision of the thinned ventricular aneurysm, the ventricle was similarly repaired with sutures tied over Teflon felt buttresses.

Cardiac bypass was re-established, after 5I minutes of circulatory arrest. Rewarming was continued for $\mathbf{7 2}$ minutes. The heart was defibrillated by a single shock.

After operation intermittent positive pressure via endotracheal tube was continued for 12 hours. The heart sounds were normal and there were no murmurs.

There was patchy basal pneumonia in the early post-operative period, and tracheostomy was performed on the 5th day to facilitate bronchial aspiration. Good progress was made and the tracheostomy tube was removed on the IIth day. Four weeks after operation he was ambulant. His chest was clear and there were no signs of failure. Maintenance therapy with digoxin, frusemide and potassium, and warfarin was continued for 6 months and then tailed off. He took his own discharge against medical advice on I January I968.

He has been seen at intervals in out-patients, though a rather erratic attender, and 22 months later he is well and working with only occasional chest pain after exertion.

The electrocardiogram recorded ro months after operation shows very little change from the pre-operative recording (Fig. I). The chest $x$-ray (Fig. 2C) and phonocardiogram (Fig. 3b) were normal.

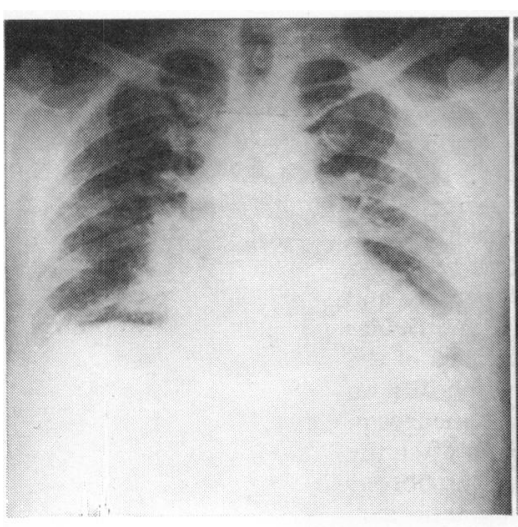

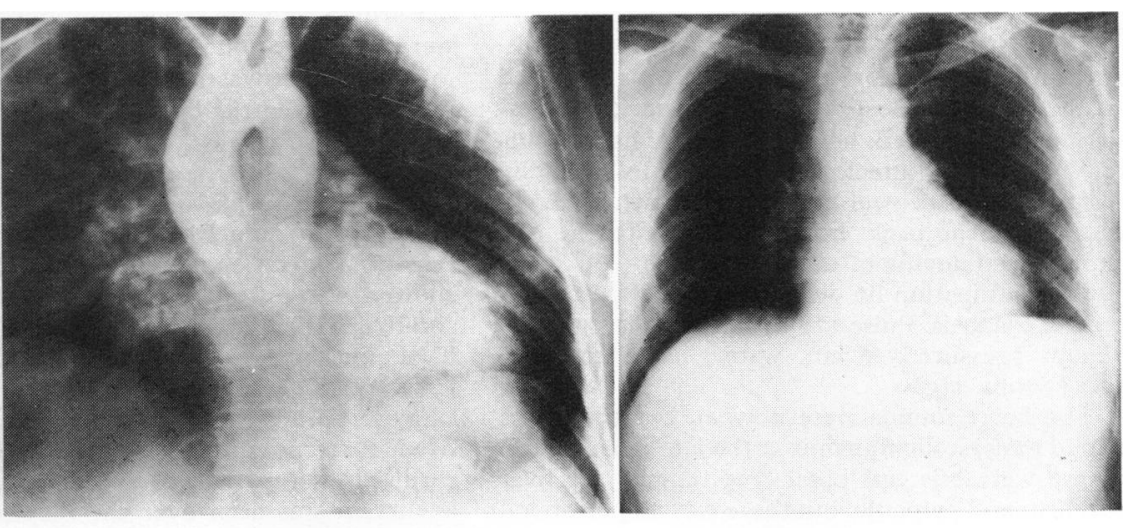

$b$ 


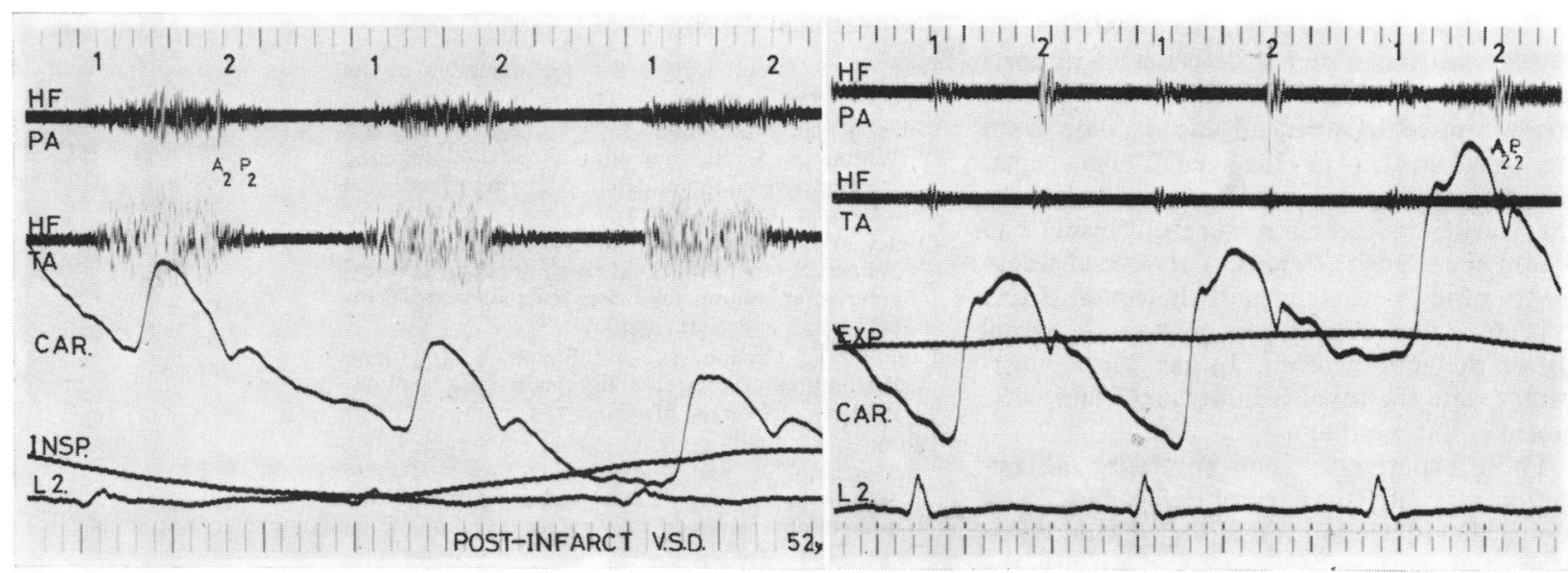

FIG. 3a Pre-operative external phonocardiogram showing the pansystolic murmur and wide splitting of the second heart sound. The time intervals are 0.20 and $0.04 \mathrm{sec} . P A$, pulmonary area; $T A$, tricuspid area; $H F$, high frequency; 1 , first heart sound; 2, second heart sound; $A_{2}$, aortic component; $P_{2}$, pulmonary component; $C A R$, external carotid pulse; INSP, inspiration; $L_{2}$, lead II of electrocardiogram.

\section{Discussion}

Collis et al. (1962) described the repair of a ventricular septal defect and left ventricular aneurysm in a man of 59. A similar procedure was performed in 1965 by Taylor et al., who corrected the two defects in a 47-year-old man. Both these operations were performed using profound hypothermia and access to the ventricular septal defect via the left ventricle, which thus permitted coincident excision of the ventricular aneurysm.

After myocardial infarction, the incidence of ventricular septal defect is about I per cent (Lee, Cardon, and Slodki, 1962) and of ventricular aneurysm about 15 per cent (Schlichter, Hellerstein, and Katz, I954). Figures for the occurrence of both defects in the same heart can be derived from Schlichter et al. (1954): of 102 patients with ventricular aneurysm, 3 per cent had a coexisting ventricular septal defect. Padhi et al. (1967) reviewed the 15 published reports of repair for acquired ventricular septal defect, and in these there was a coexisting ventricular aneurysm in 6 of the 15 hearts. Only in the two cases cited above was the repair recorded of both lesions with approach from the left ventricle. In the other 13 cases approach was from the right ventricle and only the septal defect was repaired.
FIG. $3 \mathrm{~b}$ Phonocardiogram recorded 10 months after operation showing normal splitting of the second heart sound and the absence of a systolic murmur, suggesting complete closure of the defect. EXP, expiration.
Acquired ventricular septal defects are as a rule situated in the lowest part of the septum. The normal surgical approach is from the right ventricle. This access is, however, not ideal, since the defect is often buried in the lattice of trabeculae on the right side of the septum.

Where a ventricular aneurysm coexists with a ventricular septal defect, there is an obvious route for access to the septum since the left ventricular myocardium is already damaged. The approach from the left which is thus afforded is ideal, first in that the septum on this side is smoother and the defect thus more easily defined, and secondly the left ventricular pressure keeps the patch in contact with the septum, whereas a patch applied from the right side may be forced away from the septum.

The present patient has remained well for two years after operation, experiencing dyspnoea only on severe exertion and being at full-time work. Collis's patient survived 5 years and 8 months, having enjoyed a full life (J. L. Collis, I969, personal communication). A necropsy showed the ventricular septum to be intact, and there was evidence of the old anterior infarct. The patient of $\mathrm{F}$. $\mathrm{H}$. Taylor (1969, personal communication) remains at work and is symptom free 5 years after operation. 
The timing of operation to repair an acquired ventricular septal defect is of importance. It is significant that the patients under review waited between 26 and 91 days from the development of the ventricular septal defect to its repair, and all survived. Results after earlier intervention were uniformly bad (Padhi et al., 1967). A period of several weeks allows time for the edges of the septal defect to become fibrous and thus enable safe suturing of the myocardium. In the initial postinfarct state the myocardium is so friable that suture is impossible.

These experiences show that surgical correction of ventricular septal defect caused by myocardial infarction is feasible and should be attempted in those patients who survive the critical period.

We wish to thank Dr. Aubrey Leatham and Mr. Charles Drew for permission to publish this case report and for their helpful advice in preparing the report.

\section{References}

Beck, C. S. (1944). Operation for aneurysm of the heart. Annals of Surgery, 120, 34 .

Collis, J. L., Mackinnon, J., Raison, J. C. A., and Whittaker, S. R. F. (1962). Repair of acquired interventricular septal defect following myocardial infarction. Lancet, 2, 172.

Cooley, D. A., Belmonte, B. A., Zeis, L. B., and Schnur, S. (1957). Surgical repair of ruptured interventricular septum following acute myocardial infarction. Surgery, 41, 930.

Lee, W. Y., Cardon, L., and Slodki, S. J. (1962). Perforation of infarcted interventricular septum. Archives of Internal Medicine, 109, 731.

Padhi, R. K., Fletcher, A. G., Dias, F., Servid, L. P., Mutalik, G. S., and Mody, S. M. S. (1967). Closure of ventricular septal defect following myocardial infarction. Archives of Surgery, 94, 168.

Schlichter, J., Hellerstein, H. K., and Katz, L. N. (1954). Aneurysm of the heart: A correlative study of 102 proved cases. Medicine, 33, 43.

Taylor, F. H., Citron, D. S., Robicsek, F., and Sanger, P. W. (1965). Simultaneous repair of ventricular septal defect and left ventricular aneurysm following myocardial infarction. Annals of Thoracic Surgery, $\mathbf{1}, 72$. 